\title{
Implementing Computer Mediated Communication in an Undergraduate Course - A Practical Experience
}

\author{
Mary Graham,Lecturer, School of Economics \\ Helen Scarborough, Lecturer, School of Economics \\ Christine Goodwin, Computer Mediated Communication Coordinator \\ Faculty of Business and Law \\ Deakin University \\ PO Box 423 Warrnambool, Vic. 3280 Australia \\ Telephone: +61355633149 \\ FAX: +61355633320 \\ E-mail: goody@deakin.edu.au
}

\begin{abstract}
Computer mediated communication (CMC) is used to foster a collaborative learning environment in a number of courses within the Faculty of Business and Law at Deakin University. This paper examines how this technology has been implemented at the undergraduate level in the School of Economics and some key issues arising from this experience are identified and discussed. Although the practical experience described has been gained from a particular discipline based initiative, the issues raised pertain to all disciplines. The potential provided by integrating technology into the curriculum is exciting, however this paper highlights some of the areas that need consideration when implementing an asynchronous learning network (ALN). No definitive guidelines have been provided, rather the way to maximise educational outcomes will only be found as experience and practical use is extended and shared within the academic community.
\end{abstract}

\section{KEYWORDS}

Asynchronous learning, Collaborative learning, Computer mediated communication, Computer conferencing, Distance learning, Economics education

\section{INTRODUCTION}

Deakin University is a leading distance education provider in Australia and has a strong focus on providing flexibility to students by reducing the constraints of time and place in the educational experience. Since 1991 computer mediated communication (CMC) has been used in a number of courses to create asynchronous learning networks (ALNs) that nurture a collaborative learning environment. This paper presents an overview of the CMC technology used by Deakin University and specifically how this technology has been implemented at the undergraduate level in the School of Economics within the Faculty of Business and Law. Although the practical experience described has been gained from a particular discipline based initiative, the issues raised pertain to all disciplines. The Faculty's experience suggests that there are significant differences between using educational technologies in small pilot projects and their use in large mainstream teaching programs. This extensive use of CMC required a reevaluation of the systems that underpin the teaching programs and support students and staff within both the Faculty and the University.

The development of the educational use of CMC within the University as a whole is described to provide the context within which the School of Economics has implemented its vision of an enhanced 
quality learning environment for students studying at a distance. This is followed by the details of the strategies employed by academic staff within the School of Economics who use CMC in their teaching to support large numbers of students within an ALN. Some key issues arising from this experience are identified and discussed.

\section{THE DEVELOPMENT OF CMC AT DEAKIN UNIVERSITY}

Deakin University was established in 1974 with a charter to provide both campus based and distance education opportunities for school leavers (dropouts) and mature age learners throughout Australia. It rapidly gained a reputation for excellence in distance education programs, delivered via high quality learning resources which promoted interactivity between learner and subject matter (interactive print, audiotape and videotape). Interaction between staff and students, and in some cases student to student, was facilitated by postal mail and telephone. While voluntary or compulsory face-to-face (FTF) residential schools, workshops and tutorials were also used, this was limited by the geographical remoteness of many distance learners. New communication technologies developed during the 1980's offered solutions to some of the pedagogical problems caused by geographical isolation. Pilot use quickly established the potential of $\mathrm{CMC}$ to enhance the learning environment for distance learners at the University.

In 1991 Deakin developed a prototype text-based menu interface to provide dial in or network access to e-mail, bulletin boards, the Library catalogue and an online book request service. Over the next two years the system was further developed and groups of academics began using electronic communication to help create a collaborative learning environment without the constraints of time and place. The Faculty of Business and Law began using CMC in its postgraduate programs in 1993. These programs already used collaborative learning pedagogy incorporating small group interaction. However the increasing disparity in the geographical location of students meant that interaction was less than optimal. The introduction of CMC significantly enhanced the educational dialogue opportunities for these students. Evaluations at Deakin and other educational institutions around the world indicated the benefits of enhanced educational and social dialogue through increased interaction between students and the opportunity to work collaboratively to build knowledge and understanding of course content [1], [2], [3], [4], [5], [6], [7], [8], [9], [10]. The inherent difficulties of using a text-based system hosted by a central computer and accessed via terminal emulation software limited its use, but the positive outcomes stimulated interest in developing a more sophisticated CMC system.

In late 1994, Deakin University received a grant from the Australian Federal Government for a group of projects to assist the University to develop the use of communication technologies in all aspects of teaching and learning. This major initiative was conducted in the period 1995 to mid 1997, with the Government funding supplemented by University resources. One of these projects was the development of a University wide CMC system known as Deakin Interchange. A suite of communications and application management software programs was selected and bundled into a package with an easy to use graphical interface, point and click functionality, icons, pull down menus and windows, which allowed access to networked services through standard TCP/IP Internet connections. The key teaching tool in the package was FirstClass ${ }^{\circledR}$, a computer conference system from SoftArc Inc., which was used to create ALNs that support both small and large groups.

The improvements in the operation and the reduction in complexity of the CMC software encouraged further expansion of the use of CMC into other Faculty of Business and Law programs. In 1996, offcampus undergraduate students in the Bachelor of Commerce program commenced using FirstClass ${ }^{\circledR}$ to participate in an active learning environment that complemented their self learning, and to use electronic 
resources to supplement the print based resources already provided by the Faculty. This undergraduate program enrols approximately 500 students each year, and first year units have approximately 300 students. Of these about half are distance learning students supported by CMC.

With the greatly increased availability and decreasing cost of Internet Service Providers (ISP) in Australia, Deakin has moved away from using a customised interface for bundled commercially available CMC applications. Offcampus students are now required to have Internet access through an ISP and are provided with the FirstClass ${ }^{\circledR}$ computer conference software and other course specific software on a CDRom called the Deakin Learning Toolkit. The computer conference software has become a key component of the Faculty of Business and Law's strategies for achieving its teaching and learning objectives. It is used in all Faculty teaching programs to provide students studying remotely, both within Australia and internationally, opportunities for greater interaction without loss of flexibility.

\section{IMPLEMENTATION IN THE SCHOOL OF ECONOMICS}

The Bachelor of Commerce degree at Deakin University is promoted for the flexibility given to students in terms of the range of major units of study students may select. However in the first year of the degree, all students are required to complete eight core units to ensure exposure to the range of commerce disciplines. Economics has two introductory units - microeconomics, taught in first semester, and macroeconomics, taught in the second semester. Off-campus students are usually studying part-time and select two units of study per semester. Most would complete the two units of economics over the two semesters within the same year.

Traditionally economics has a reputation of being difficult for students [11]. This is particularly the case for first year economics as demonstrated by the around 50\% fail rate, with only one quarter of Australian universities having a fail rate below 25\% [12]. Many factors contribute to this such as the introduction of a new vocabulary specific to economics, the requirement of students to possess abstract and technical thinking skills and the mixed skill levels ranging from those with pre-tertiary studies in economics and mathematics to those without [11]. The staff in the School of Economics faced the challenge of adapting the undergraduate units to maximise the potential benefits of CMC and create a richer learning experience that addressed some of these difficulties. Collaborative learning as a problem-solving device was incorporated in the pedagogy adopted. ALNs are an effective medium to provide the social aspect of learning emphasised by collaborative learning. Through formulating ideas in their words, and receiving feedback and evaluation from peers, students' knowledge, thinking skills and meanings are socially constructed [13].

Each off-campus student was provided with the choice of learning using the traditional method, involving printed course notes, phone contact with staff, assessment via assignments (which are returned with comments) and a final exam; or learning using the First Class conferencing system to provide, in addition to the above, communication with both other students and staff. The use of computer conferencing also allowed for some assessment dependent on the completion of small group tutorial work. It was deemed necessary to offer a choice, as making it compulsory for all to have access to a computer and the Internet could be challenged on equity grounds, although it can be argued that computer literacy is an essential skill for a commerce graduate.

In 1996, the first year of implementation of the program, 181 students studying macroeconomics were given the option of participating in the online program. Fifty students initially registered their desire to be connected, however, due to course withdrawals and difficulties with the technology, the final number was 39 students. This take up rate for participation in the online program, at $21.5 \%$, was lower than 
anticipated. The numbers participating in subsequent years have increased but not as rapidly as hoped. The participation rate in the second year of the program was $26 \%$.

To develop the opportunity for online communication and collaboration activity, students were allocated to tutorial groups of 6 students as they connected to the system at the beginning of the semester. Group formation was independent of factors such as geographic situation, previous experience, and common areas of study. Students could belong to a different group for each unit of study. At no time during the course delivery, did students experience University organised FTF interactions.

The aim was for students to experience increased support, motivation and learning through actively solving economic problems collaboratively. Collaborative or group learning is premised upon a learnercentred model that treats the learner as an active participant who construes knowledge from a wide range of experiences, information sources and interaction with others [14], [15]. Students in their syndicate groups could discuss the areas with which they faced difficulties.

The main tutorial activity centred on group work with exercises operating on a two-week cycle. The questions set in the exercises resembled as closely as possible the work being completed by on-campus students in tutorial groups. As all students complete the same final exam, equity in terms of the material completed was an objective. As each new exercise was posted, the previous group effort was posted to the tutor for comments and/or assessment. Each student was instructed to take responsibility for one question and after researching the topic, post his/her answer on the group tutorial board. Comments and discussion were encouraged to promote interdependence and increase the level of support and motivation experienced by students. One member of the group was required to collate final answers and submit the group's exercise to the tutor. Group ownership of the exercise was encouraged as each member of the group shared the same mark for the exercise yet individual accountability was also present, since students ultimately held responsibility for the final format of their assigned question. The key elements identified in the literature as promoting collaborative learning, namely interdependence, individual accountability, interpersonal and group skills and group processing were encouraged.

Each group was assigned a tutor whose role was complementary to both the group and the individual student's work. The syndicate group became the focus of learning with the tutor, no longer at the centre of the learning process, initiating active group approaches to learning and assisting individuals to come to terms with the theory. The isolation many off-campus students experience was reduced and student-staff communication was enhanced. To provide a genuinely flexible learning environment, communication was asynchronous, whereby users could participate at a time and place convenient to them and appropriate to the task. By leaving messages and responding to other students' questions, a learning environment independent of time and place was created as participants could respond immediately or they could elect to respond after taking time to reflect and compose a response thoughtfully. The cumulative record of message contributions provided greater potential for reflective thought, analysis and review of earlier contributions than participation in FTF seminars. This experience supports the claim of Kaye [5] that although the para-linguistic cues of FTF or telephone communication are missing, the medium does offer greater communication richness than the more familiar forms of textual communication used by groups.

Communication could occur at three levels: (1) a general level within the Bachelor of Commerce (BCom) conference, open to all registered users within the Faculty; (2) a more discipline-specific level within the unit of study; and (3) the accompanying tutorial group. Figure 1 illustrates the entry screen for the BCom program and shows the subconferences used for academic, administrative and social purposes. All students enrolled in the BCom program can use this area, thus providing a context for their study of 
individual units. Although this picture of the screen was taken recently, it represents the CMC environment in use during the time period reported in this paper.

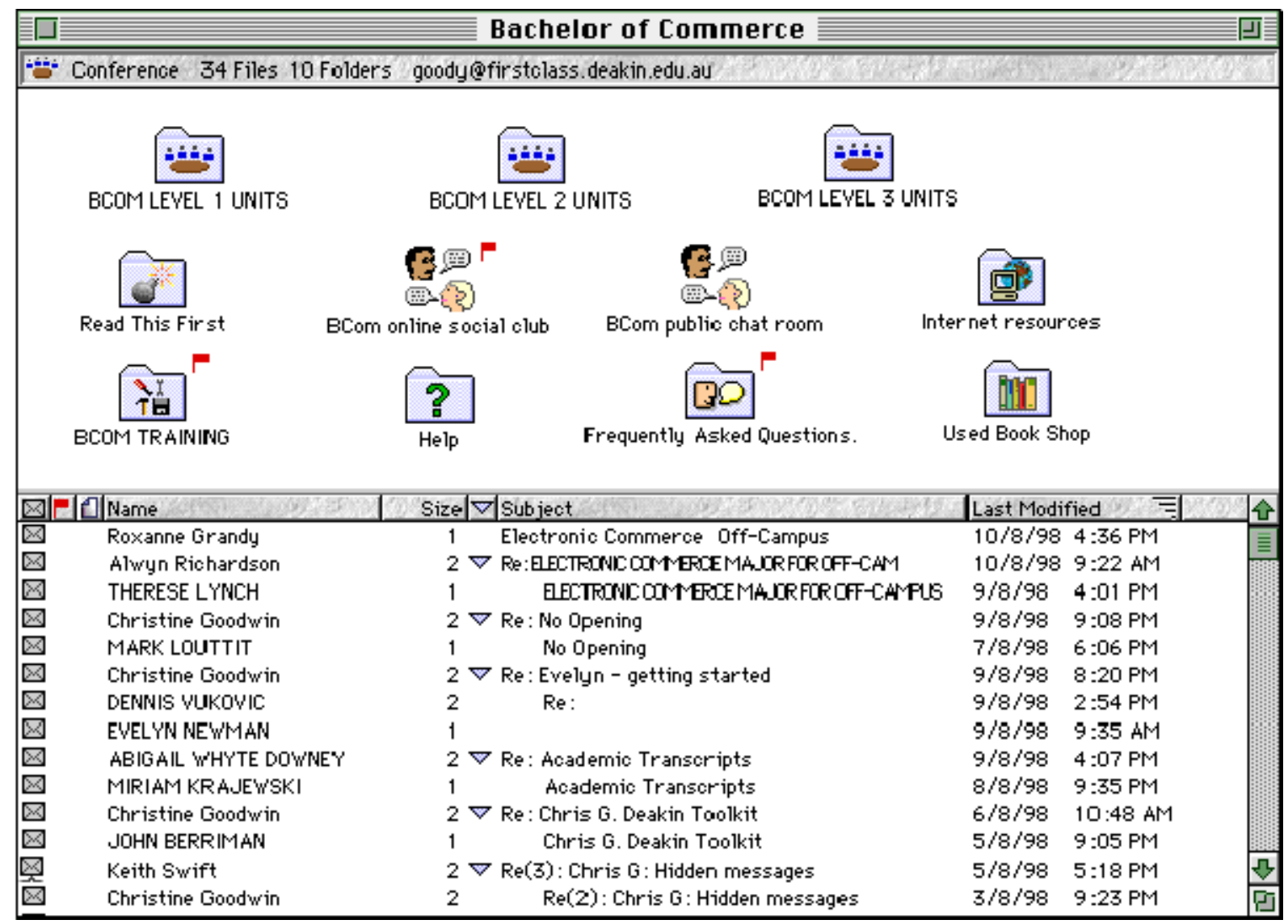

Figure 1. The Bachelor of Commerce Computer Conference Environment.

The unit conference replicates an open general classroom with both administrative and teaching information conveyed through this avenue. In Figure 2 the lower section of the window displays messages showing interaction (student-student, student-staff) within the larger student body studying Macroeconomics. The interactions range from a discussion of key concepts contained within the unit of study to administrative matters relating to assessment or additional references. The upper section contains subconferences which have specific purposes as indicated by their captions. At commencement of the semester, students leave a message in the MEE102 Sign On folder indicating their willingness to be involved in the small group tutorial program. The Resources folder contains additional reference material, including relevant Web sites. The fortnightly tutorial exercises and their solutions are located within the Assessment folder. Important administrative announcements are posted within the MEE102 Noticeboard to ensure they are not lost in the volume of messages created by general discussion. The final folder, Current Issues, is used to stimulate discussion of the application of the theory being studied to current economic developments both nationally and internationally. 


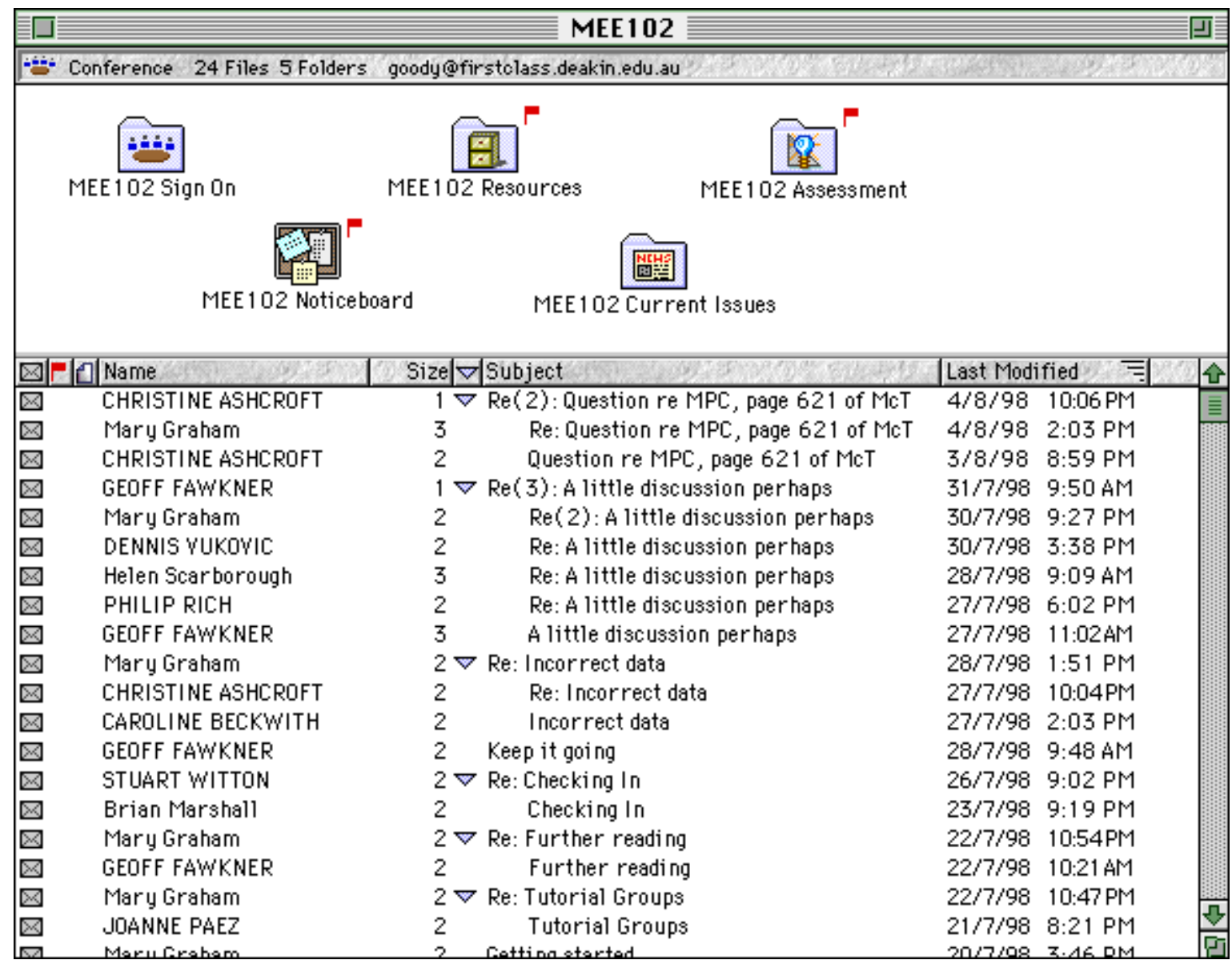

Figure 2. The MEE102 Macroeconomics Unit Conference Showing Large Group Interaction and Specific Purpose Subconferences.

The focus of the tutorial group, as shown in Figure 3, was on the assessable fortnightly exercises with access limited to the group members and the tutor. Discussion centred on the relevant answers to the questions, individual responsibilities and the collation of the group's submission. Much of the interaction was student centred peer review of initial responses to the task, with the modifications reflecting input from other group members forming the final group response. The tutor monitored this interaction and when necessary, provided direction. Following the final group submission the tutor provided feedback and awarded a grade. 


\begin{tabular}{|c|c|c|c|c|c|c|c|c|}
\hline 柜士 & & & (" & MEE & 02 - Jeuons & & & 可 \\
\hline$E: 0$ & 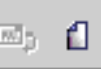 & 雷 耑 品 & 防 仿 & d) 容 & 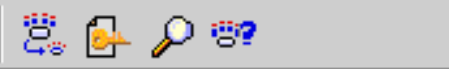 & & & \\
\hline$\therefore \mathrm{C}$ & nference & 227 Files 0 Fold & rs FC Deak & ins First & lass : goody & & & \\
\hline 国 & Name & & Size $\mid \nabla$ & Subject & & Last Modifi & fied & 노 \\
\hline बi & Mary $\mathrm{G}$ & ham & $2 \nabla$ & Re: Sen & eester End & $27 / 10 / 98$ & $11: 00 \mathrm{PM}$ & \\
\hline 国 & PHILIP & & 1 & & nester End & $27 / 10 / 98$ & $9: 44 \mathrm{FM}$ & \\
\hline 雨 & Mary $\mathrm{G}$ & ham & $2 \nabla$ & Re: Gro & up Submission, Tutorial 6 : Comment & $27 / 10 / 98$ & $10: 56 \mathrm{PM}$ & \\
\hline 田 & QUAN N & UYEN & 1 & & Group Submission, Tutorial 6 & $27 / 10 / 98$ & $6: 24 \mathrm{PM}$ & \\
\hline बr & PHILIP & & 8 & & up Submission, Tutorial 6 & $25 / 10 / 98$ & $8: 28 \mathrm{PM}$ & \\
\hline 电 & QUAN N & UYEN & $1 \nabla$ & Re: End & of Semester & $27 / 10 / 98$ & $6: 20 \mathrm{PM}$ & \\
\hline बr & GREGOF & GRAY & 2 & & of Semester & $26 / 10 / 98$ & $7: 27 \mathrm{PM}$ & \\
\hline 四 & $J O H N S$ & HOLZ & $1 \nabla$ & Re: Tut & $6,0.5$ & $24 / 10 / 98$ & $5: 55$ PM & \\
\hline 四 & QUÁN N. & UYEN & 3 & & $6,0.5$ & $23 / 10 / 98$ & $12: 50 \mathrm{AM}$ & \\
\hline 四 & $J O H N S$ & HOLZ & $1 \nabla$ & Re: Tut & $6,0.5$ & $22 / 10 / 98$ & $7: 24 \mathrm{PM}$ & \\
\hline बr & QUAN N & UYEN & 1 & & e $6,0.5$ & $21 / 10 / 98$ & $10: 21 \mathrm{PM}$ & \\
\hline 四 & $J O H N S$ & HOLZ & $1 \nabla$ & Re: Pos & ting Group Submission & $22 / 10 / 98$ & $7: 22 \mathrm{PM}$ & \\
\hline 国 & PHILIP & & 1 & & ting Group Submission & $21 / 10 / 98$ & $3: 19 \mathrm{PM}$ & \\
\hline 四 & JOHN S & HOLZ & $1 \nabla$ & Re: Tut & 6. - Q. 4. Response & $2 / 10 / 98$ & $7: 19 \mathrm{PM}$ & \\
\hline 四 & GREGOF & GRAY & 2 & & 6. - Q. 4. Response & $21 / 10 / 98$ & $1: 31 \mathrm{PM}$ & \\
\hline 国 & GREGOF & GRAY & 2 & Tut 6 - & Q.6. Response & $21 / 10 / 98$ & $1: 44$ PM & \\
\hline 田 & PHILIP & & $1 \nabla$ & Re: Tut & rial 6. - Question 2 & $19 / 10 / 98$ & $10: 13 \mathrm{PM}$ & \\
\hline 田 & GREGOF & GRAY & 2 & & orial 6 - Question 2 & $19 / 10 / 98$ & $11: 02$ AM & \\
\hline 雨 & PHILIP & & $1 \nabla$ & Re: Tut & rial \#6, Q4. Response & $19 / 10 / 98$ & $10: 11 \mathrm{PM}$ & \\
\hline 田 & $J O H N S$ & HOLZ & 4 & & orial \#6, Q4. Response & $18 / 10 / 98$ & $12: 11 \mathrm{PM}$ & \\
\hline 四 & Mary G & aham & $3 \nabla$ & Re: Tut & orial \#5 - Group submission & $19 / 10 / 98$ & $7: 11$ AM & \\
\hline 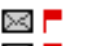 & Mary $\mathrm{G}$ & aham & 1 & & Tutorial \#5 - Group submission & $11 / 10 / 98$ & $10: 57 \mathrm{PM}$ & \\
\hline 国 & JOHN S & HOLZ & 8 & & orial \#5 - Group submission & $11 / 10 / 98$ & $3: 03 \mathrm{PM}$ & \\
\hline 四 & PHILIP & & 3 & Tute 6 , & Q6-Suggested Answer & $14 / 10 / 98$ & $11: 02 \mathrm{PM}$ & \\
\hline 田 & QUAN N & UYEN & 1 & Tute 6 , & Q. 5 & $14 / 10 / 98$ & $9: 10 \mathrm{PM}$ & \\
\hline 雨 & PHILIP & & 1 & Tut 6 , & uestion 6 & $13 / 10 / 98$ & $8: 54 \mathrm{PM}$ & \\
\hline बr & GREGOF & GRAY & 1 & Tutoria & 6 - Question 2 & $12 / 10 / 98$ & $8: 35 \mathrm{PM}$ & $\sqrt{3}$ \\
\hline 227 It & & & & & & & & 口] \\
\hline
\end{tabular}

Figure 3. A Typical Tutorial Group Conference Within the MEE102 Macroeconomics Unit.

This is the environment within which the small group collaborative learning exercises are conducted with a tutor acting as mentor.

\section{KEY ISSUES}

From the experience in developing an educational program for undergraduates within the School of Economics, the following key issues have been identified as crucial areas to be considered when adopting CMC technology.

\section{A. Access}

In an online learning environment, access is critical to success. All effort expended in implementing CMC in a teaching program will be wasted if students are unable to "attend the classroom." The technical nature of ALNs and the necessity to operate a computer and modem presented a barrier to many students in this program. This experience highlighted that the processes involved in both initial and ongoing access must be easy and engender confidence in students who may have varying levels of experience with computers and online communication.

It is also critical that ongoing access throughout the period of study remains easy and reliable. When computer mediated systems are introduced as part of a teaching program they create an expectation that the online services will be available whenever the student or staff member wants to use them. The 
infrastructures that supported the use of CMC in this case included local telecommunications networks, the Internet Service Providers who supply access to the national and international infrastructure of the Internet itself, and the network infrastructure at Deakin University. A failure in any part of this complex system resulted in frustration and, in some cases, unnecessary concern on the part of students that they were "doing something wrong" when the problem may not have been within their control. Repeated failures lead to a perpetuation of the isolation that is often experienced by students studying remotely so that one of the key benefits of using CMC in remote learning situations is negated. When the assessment of student learning requires the use of CMC to facilitate collaborative assignments or online submission of work, a great deal of stress and even panic can be created by difficult or unreliable access.

Academic staff must be prepared to cope with these emotions in their students and have readily available support from technical staff to provide information to the users of the CMC technology. Excellent documentation, easy to install software and a timely and effective response from the Help Desk are all essential to ensure access and the smooth operation of the teaching medium. Institutional commitment of resources to establish and maintain the underlying network infrastructure within the institution is also critical.

\section{B. Group Dynamics}

The online environment offers the opportunity for students to share with others their thinking, interpretations, and reactions to the ideas that are being presented. For distance education students, in particular, this is a rich learning environment when compared with the isolation they traditionally experienced. Students differed in their approaches to this new learning environment where there are fewer social cues to shape participation, hence, the extent of interdependence and cooperation of each group varied. Cooperation was evident in some groups, while in others, students took responsibility for the question assigned to them and did not collaborate. It became clear that many students are not comfortable with group work, particularly when incorporating the sharing of assessment. However, the linking of the group tasks to the assessment process was critical, as when the tasks were not assessable the interaction between and commitment of group members diminished. To some extent this reflects rational choices by mature age students who may be attempting to balance the conflicting demands of employment, family and study. The general maturity of the students was also evident in some aspects of the group operations and communications.

Tutors observed the group discussions and used their discretion regarding intervention. As with FTF group work, there were problems with those seeking to dominate and those who watched without contributing. The advantage of ALNs is the visibility of each group member's contribution for the group participants and the tutor. Asynchronous communication means that the extrovert personalities may leave many more messages than quieter members, however, they cannot dominate completely as in a FTF situation. Quieter members still had the opportunity to contribute. This supports research by Straus [16] suggesting that the equalisation effect is caused by the ability to participate simultaneously. Nonparticipants were either students who read messages but did not contribute, or those who failed to have regular access. The administrative functions of FirstClass enable tutors to determine when a student last connected and if they have read the messages. This was used to determine appropriate intervention. Strategies for dealing with the non-participants commenced with messages of encouragement within the group environment reinforcing the need to connect regularly and leave a message to register their presence. If this did not provoke a response, personal contact was made by private e-mail or telephone. Students who elected to continue with the online program but did not contribute to the group effort forfeited the marks allocated to this aspect of assessment. Although this was a time consuming process in the first weeks of semester, the ongoing function of the groups benefited greatly from this early intervention. 
These observations suggest the following factors should be considered when seeking to maximise the educational discourse in CMC centred collaborative learning groups. Firstly, the composition and size of the groups plays a significant role in the group dynamics. Care needs to be taken to make the groups small enough to avoid free riding while maintaining sufficient numbers to ensure a critical mass for active discussion. The method of group formation also impacts the ensuing dynamics. In the first year of implementation, students were randomly allocated to groups following enrolment. However technical difficulties resulted in a significant number not connecting, leaving many groups dysfunctional due to sub-optimal numbers. In subsequent semesters, students were asked to register their interest in participating in the online tutorials by leaving a message in a "signing on" folder. Groups were then allocated sequentially according to this list. With some exceptions, the students who were most keen signed on earlier and therefore were grouped together. Hence a notional form of streaming had been inadvertently incorporated in the group formation process. This was an administratively efficient method of group formation, but it ignores attributes such as gender, age, ethnicity, educational and work backgrounds that may influence educational outcomes. While we felt these attributes would not significantly impact student learning or experience in this program, they may need consideration when forming groups in other circumstances.

As with all communication, it takes time to develop a degree of familiarity. The fact that students in the Bachelor of Commerce program belonged to a different group for each unit studied, and only met fellow students via their online communication, seemed to make communication within the group more constrained when compared to the course-long syndicates established in other programs. Our experience has not shown clearly whether an introductory FTF meeting is of benefit to the dynamics of the group. However, it is apparent that in the absence of such an activity, it is necessary to incorporate carefully planned introductory, non-academic tasks to allow members to feel comfortable with both the group interactions and their online communication processes.

Clear guidelines and expectations for participation need to be developed and readily available. Individual and group responsibilities need to be stated clearly at the beginning to ensure maximum participation and contribution. The asynchronous feature allows the learner more time to think about his/her contribution than in direct and spontaneous responses. But by reducing the pressure to respond, it is easier for the student to drop out of the group. Contributions from students and staff need to be regular and timely and provide feedback to individual members in a positive and constructive manner.

\section{Course Content and the Medium}

Staff faced the challenge of adapting course content to this medium, the difficulty of which varied between disciplines and level of study. There was a need to adapt teaching strategies to benefit from the extended possibilities technology allows. As Garrison [17] states, while computer conferencing lends itself to collaborative and constructivist pedagogies, this does not happen by simply making the technology available. Collaborative learning emphasises cooperative efforts and common inquiry as the basis for learning and the issue of whether this approach is suitable to the teaching of introductory economics became apparent. Collaboration is more than simply exchanging information or passing on instructions. Meaningful collaborative learning creates "added value" and new understandings amongst the members of each group [5]. The tutorial questions tended to be fairly theoretical and conceptual reflecting the nature of the course material. First year students were more focused on how well they had understood and interpreted the material given in the text. It was difficult to stimulate students to express differing views and generate discussion but they appreciated peer explanation of the concepts, with many acknowledging that it gave them a better understanding. As one student commented: 
"I will read the other answers because it helps me understand this subject better. I think the answers from the group have helped me a lot. It saves on reading long passages of text and gives me a good overview."

For another student:

..the communication aspect is important in these tutes[tutorials].. I have had a look through the question you are doing and have recognised the following connections between East Asia and Japan.....

The online environment provided the opportunity to build on the resources available to students. Discussions were integrated with the text and study guide provided, so that students learnt by discussing concepts rather than just reading. Students benefited from explaining to their peers their understanding of a particular concept or why their interpretation differed from others in the group. Active participation encouraged learning, as highlighted by the following comment by a student:

I find participating in all questions (not only the one I have to answer) extends my understanding of the topic. By considering someone else's answer, I can often see where their perceptions assist me in seeing topics from another viewpoint. I would prefer a larger proportion of our assessment was conducted using these tutorials.

Current and relevant articles from newspapers and journals were scanned and posted to the conference to add value to the existing resources. For example, the unit conference had a folder set aside for discussion of current issues and their relevance to the theory under study. The development of WWW hyperlinks in the conferencing facility, although not available in the time period under study, now provides the opportunity of incorporating other resources for students allowing them to explore areas of interest. Using multimedia resources within an educational framework can easily extend material relevant for particular topics. Reference materials, for example current articles scanned and posted on the conference service or links to appropriate web addresses, can be easily updated and incorporated into a unit at any time. This is an exciting development for off campus study where traditionally the lead time required for production of high quality printed material has prohibited dynamic change.

\section{Workload}

The issue of workload is of concern to both staff and students. Of those students who dropped out of the online program (1996-22\%, 1997-13\%) some blamed difficulty in accessing the system while for others the combination of study, work and family commitments proved too much. The requirement to be responsible for part of an exercise every fortnight was daunting as it required students to keep up to date with their study and initially some students claimed that this reduced the flexibility expected from distance education. But flexibility is still possible as all communication is asynchronous and students can contribute to the group discussion at a convenient time.

Students need to consider that by adopting a more structured approach to their study, much of the stress experienced towards the end of a semester can be reduced. By the end of the semester, many appreciated the benefits of a structured approach to the pace of study. Care however is needed to balance the many commitments of students, particularly mature aged, full-time members of the workforce. As one student commented:

The fortnightly tutorial makes you keep up to date as much as possible as you feel you have a responsibility to the others in the group, without this I know it is easy to get behind with all the pressures of everyday life such as jobs, families... 
Purposeful, regular and directed study should not be easily disregarded and must be acknowledged and included as part of their overall assessment. It need not be a large part, but should be adequate to recognise the time devoted by students to undertake the tasks fully.

From the point of view of staff, the student inquiries can often be more efficiently dealt with in that answering one query can cover many. Alternatively, the asynchronous feature allows individual students to be given assistance if necessary without detracting from the group activity. However, the easy access to staff increases the student expectation for contact and allowance has to be made for this in determining teaching loads. The flexibility offered by the system is also available to staff who should advise students of an appropriate timeframe in which to expect a response to their inquiries.

An issue that is of concern to staff developing the program is the low take up rate by students. Within the School of Economics, students have been presented with an alternative to the online program and assessment. For various reasons many students have opted to remain with the traditional written assessment. This may reflect their fear of flexibility being reduced if they commit themselves to being part of a group, or their preference for individual work as opposed to group work, or the fact that they are comfortable with what has been tried and proven in the past. However, it is felt that as long as a choice is given we may find that although the delivery of the course material to off-campus students is greatly enhanced with electronic delivery, staff workload increases considerably.

The adaptation of pedagogy to this style of teaching requires thought and a good deal of time to reengineer the curriculum and develop extensive access to resources. It is important that these needs be given adequate recognition by the institution. However, our experience is that high student contact hours, increasing administrative responsibilities and research demands leave insufficient time to reengineer and to develop extensive access to resources. In addition, the current funding environment within the Australian higher education sector leaves little opportunity for universities to be flexible in the provision of resources required to facilitate this need.

\section{E. Staff Training}

Academic staff using the system required guidance while retaining the responsibility to deliver the unit and determine the most appropriate use of CMC in their teaching and learning program. Staff differed greatly in their knowledge of CMC and the problem this presented was compounded by the physical difficulties of a multi-campus university. The early users were highly motivated with a great deal of enthusiasm to learn by themselves while later experiences have shown that staff need to be persuaded of the value of using CMC in their teaching in order to devote precious time to learning new skills. The principle of learning by doing was applied from the introduction of the system. While this was adequate initially, professional development and systematic training on innovatively using the system for subject content and assessment has proved necessary as the use of CMC becomes an integral part of our teaching programs.

Since familiarity with a particular technology increases the perception of its possible use in the future there is a need for academic staff to have increased exposure to the use of CMC technologies in a teaching context which involves hands-on experience. The formation of a favourable or unfavourable attitude toward using a technology is most influenced by informal communication with colleagues who have used the technology [18]. Hands-on experience and trial use increase the understanding of the benefits and possibilities of CMC technologies in the teaching and learning environment, and reduce the uncertainty involved in making a decision to use them. 
At Deakin, the Centre provided formal workshops on basic operational aspects of the software for Academic Development. Further operational knowledge of the software tools can be given on a 'just in time' basis followed by instruction on more advanced features of the software as staff become more proficient. The Computer Mediated Communication Coordinator within the Faculty gave such individualised advice and training. The more complex issues of achieving effective staff and student interaction, managing and evaluating the ALN, and developing pedagogy to suit the online environment have been addressed by the Faculty in an experiential collaborative learning online program. Faculty leaders endorsed this program and strongly encouraged all staff to participate. The five-week program was conducted asynchronously in a learning environment that closely replicated that in which they would be teaching. It is envisaged that such professional development programs which simulate the teaching context in a realistic way and allow creative "learning by doing" in a non-threatening environment will assist academics to understand the potential benefits of using CMC in their teaching.

A broad range of skills is required to effectively use CMC in teaching. As use of CMC evolves there is a need for staff to explore the educational design and development of online teaching environments and to enhance their communication and facilitation/moderation skills. The availability of time to do this effectively is raised repeatedly when staff are asked what are the major challenges they face in keeping up with change in information technology:

"Finding the time, firstly to get to workshops, etc, and then to have time to follow up trying out what has been learnt."

"Time to learn to use it and flexible availability of support personnel to teach the use. It is more important in the University's eyes to get on with research."

"Finding time to be aware of what is available, how to incorporate it in my teaching and having the time to tailor it for my use."

It is difficult for staff with already heavy teaching and research workloads to allocate sufficient time to acquire or enhance these higher level skills, but the neglect of this will lead to inappropriate and unstimulating uses of CMC. The broad nature of the staff training necessitates institutional commitment to providing adequate resources for training programs and teaching relief while staff acquire new and enhanced skills. At Deakin there is now recognition of the importance of structured training programs, but the question of adequate teaching relief remains unresolved.

\section{F. Evaluation}

The evaluation of educational programs is often difficult and analysis of the effectiveness of using CMC is no exception. In essence, there are three issues requiring evaluation: student learning outcomes, student access, and cost factors to both the student and the university. It is the first of these which is the responsibility of academic staff and hence where our emphasis in this paper lies.

The distinction between the evaluation of the effectiveness of the learning and the functionality of the medium is crucial as access statistics and the number of messages do not indicate student learning. The distinction is sometimes difficult to determine as access to the technology is an integral component of the success of the educational program. Care needs to be taken not to place too much emphasis on quantifiable outcomes such as the marks gained in the subject or the number of times students used the system. It is the content of the messages that display students' understanding of the theory, and qualitative feedback from students which indicates the value of their learning experience. With students' learning outcomes, benefits are both tangible, such as improved understanding of the material, and 
intangible, such as a sense of belonging to a group. While marks indicate ability to conceptualise introductory theory, they may not reflect the educational process through which students have progressed.

The problems associated with quantitative evaluation are compounded by the difficulty in measuring and controlling for the myriad of factors that influence student learning outcomes. Direct comparison of grades achieved by students participating in the online program, as opposed to those who did not, is of limited use due to the discrepancy between the two pedagogical regimes. In addition, the establishment of a randomly selected control group is not possible and this creates inherent self selection biases. As an education provider, equitable opportunities must be provided to all students and therefore a control group can only be established through self selection.

In many respects qualitative analysis will be more enlightening than quantitative data. The ease of communication with distance students using CMC allows for effective qualitative evaluation through a continual process of inquiry as to the students' progress through the unit. This can be achieved through a specific conference where students can record comments on issues such as assessment processes and unit content. The experiences of staff and students will also provide different perspectives to the learning outcomes. Care needs to be taken to separate the evaluation of the unit content from evaluation of the method of study.

A further complication to the evaluation process is the role of attitudes in students' learning outcomes and the difficulties associated with measuring attitudes and changes in attitudes. Yet, this aspect is particularly significant with respect to the introduction of new technology and an innovative educational environment. One student commented,

I started the semester feeling very hesitant but I, like many others, have found this medium to be extremely useful.... I have found it very beneficial in that I can get help online, discuss work and socialise online and I can also work within a group.

Evaluation needs to be an integral part of the establishment of a learning program, particularly as the use of technology becomes widespread. The costs and effort involved in implementation, and the financial pressures being applied to tertiary education, indicate that carefully considered evaluation is required to ensure quality and demonstrate accountability.

\section{CONCLUSION}

The introduction of CMC technology presents a challenging new stage in the provision of tertiary education. This paper has identified and discussed some of the issues arising from the authors' practical experience with integrating the technology into a learning program. The potential it provides is exciting, however the issues raised highlight some of the areas that need further consideration. No definitive guidelines have been provided. Rather the way to maximise educational outcomes will only be found as experience and practical use is extended and shared within the academic community. To optimise the potential provided by the pace of technological change, teachers in all disciplines must be prepared to take up the challenge to examine, and if necessary change, what they teach and how they teach.

\section{REFERENCES}

1. Harasim, L., Online Education: A New Domain. In: Mason, R., and Kaye, A., (Eds.), Mindweave: Communication, Computers and Distance Education, Oxford: Pergamon Press, 1989. 
2. Mason, R. and Kaye, A., Toward a New Paradigm for Distance Education. In: Harasim, L., (Ed.), Online Education: Perspectives on a New Environment, New York: Praeger, 15-38, 1990.

3. Bates, A.W., Third Generation Distance Education: The Challenge of New Technology, Research in Distance Education, 3 (2), 1991.

4. D'Souza, P.V., The Use of Electronic Mail as an Instruction Aid: An Exploratory Study, Journal of ComputerBased Instruction, 18 (3), 106-110, 1991.

5. Kaye, A.R., Learning Together Apart. In: Kaye, A.R., (Ed.), Collaborative Learning Through Computer Conferencing, London: Springer-Verlag, 1991.

6. Boston, R., Remote Delivery of Instruction via the PC and Modem, The American Journal of Distance Education, 6 (3), 45-57, 1992.

7. Rich, M., The Use of Electronic Mail and Conferencing Systems in Teaching Management Students, Working Paper Series, London City University Business School, 1992.

8. Lewis, C.T., and Hedegaard, T., Online Education: Issues and Some Answers, T.H.E Journal (Technological Horizons in Education), 20 (9), 68-71, 1993.

9. Hiltz, S.R.,. The Virtual Classroom: Learning Without Limits via Computer Networks, Norwood, NJ: Ablex Publishing Corporation, 1994.

10. Mason, R., and Kaye, A., Mindweave: Communication, Computers and Distance Education, Oxford: Pergamon Press, 1994.

11. Cowie, J., Findlay, C., Round D., and Shanahan, M., Bodies and Mind - Achieving Teaching Quality in Economics: The Adelaide Method, HERSDA Annual Conference, 1994.

12. Kniest, P., Australian Survey of First Year Economics Courses: Course Organisation, Structure and Staffing, Teaching First Year Economics at Australian Universities, The University of Newcastle, Department of Economics, 1993.

13. Harasim, L., Hiltz, S.R., Teles, L., and Turnoff, M., Learning Networks: A field guide to teaching and learning online, Cambridge: The MIT Press, 1995.

14. Harasim, L., Online Education: An Environment for Collaboration and Intellectual Amplification. In: Harisim, L., (Ed.), Online Education: Perspectives on a New Environment, New York: Praeger, 1990.

15. Laurillard, D., Rethinking University Teaching: A Framework for the Effective Use of Educational Technology, London: Routledge, 1993.

16. Straus, S.G., Getting a Clue: The Effects of Communication Media and Information Distribution on Participation and Performance in Computer Mediated and Face-to-Face Groups, Small Group Research, 27 (1), 115-142, 1996.

17. Garrison, R.D., Computer Conferencing: the Post Industrial Age of Distance Education, Open Learning, June 1997, 3-11, 1997.

18. Brancheau, J.C., and Wetherbe, J.C., Understanding Innovation Diffusion Helps Boost Acceptance Rates of New Technology, Chief Information Officer Journal, Fall 1989, 23-31, 1989.

\section{ACKNOWLEDGMENTS}

The authors wish to acknowledge the helpful comments of the participants at the ISETA 1997 conference held in Fredericton, New Brunswick, Canada. 